Philosophy and Phenomenological Research

Vol. LXXI, No. 3, November 2005

\title{
Semantic Pathology and the Open Pair
}

JAMES A. WOODBRIDGE

University of Nevada, Las Vegas

BRADLEY ARMOUR-GARB

University at Albany, SUNY

\section{Introduction}

In Vagueness and Contradiction (2001), Roy Sorensen defends and extends his epistemic account of vagueness. In the process, he appeals to connections between vagueness and semantic paradox. These appeals come mainly in Chapter 11, where Sorensen offers a solution to what he calls the no-no paradox-a "neglected cousin" of the more famous liar-and attempts to use this solution as a precedent for an epistemic account of the sorites paradox. This strategy is problematic for Sorensen's project, however, since, as we establish below, he fails to resolve the semantic pathology of the no-no paradox.

The relevant case of semantic pathology — that is, of a putative misfiring of our familiar semantic concepts - arises in what we call the open pair:

(1) (2) is false

(2) (1) is false. ${ }^{1}$

Central to cases of semantic pathology is a resistance to semantic characterization that arises out of the ordinary operation of the semantic predicates. It is widely appreciated that this phenomenon bifurcates into two symptoms: inconsistency, as manifested in liar sentences (e.g., 'This sentence is false'), and indeterminacy, as manifested in truth-teller sentences (e.g., 'This sentence is true'). Given familiar logical and semantic assumptions, the open pair is semantically pathological, as these sentences exhibit the telltale

The original source for this case is Jean Buridan's Eighth Sophism from Chapter 8 of Sophismata. See Hughes (1982). Having worked on and developed our own label for this case before becoming aware of Sorensen's, we prefer ours, in part because, as we show presently, while the open pair is pathological, it is not paradoxical because it does not force inconsistency. 
resistance to semantic characterization, arising from the standard operation of the semantic predicate 'false'.

In fact, this case will manifest either symptom of resistance, depending on what we say about these sentences. (1) and (2) together yield inconsistency, if we ascribe them matching truth-values. But inconsistency is not inevitable here; we can avoid it by claiming one of these sentences is true and the other false. This, however, yields indeterminacy, as there are two ways of ascribing divergent truth-values, and nothing appears to favor one over the other. Thus, if unresolved, these sentences manifest one or the other symptom of semantic pathology.

Sorensen offers his solution to the open pair as an improvement on one Laurence Goldstein (1992) has presented. Goldstein attempts to resolve the semantic pathology of (1) and (2) by arguing that they are gappy, i.e., without truth-values. Sorensen, by contrast, claiming that a good theory should minimize "truth-bearer illusions" (p. 183), ${ }^{2}$ maintains that (1) and (2) have determinate, consistent truth-values, though which truth-value each sentence has is epistemically indeterminate. These circumstances are the result of a truthmaker, as opposed to a truth-value, gap, the obtaining of which deprives us of access to either sentence's truth-value.

While Sorensen's approach skirts a fundamental objection to Goldstein's account, neither of these attempted 'gappist' solutions resolves the problem posed by the open pair. In each case, principles adduced to deal with this case of semantic pathology are undermined by a revenge problem that thwart the consistentist's attempt to resolve it. While this bodes ill for consistent approaches to semantic pathology, as we establish below, contra Priest's (2004) claim that he can inconsistently resolve the open pair, the very same revenge problems that undermined the accounts of Goldstein and Sorensen do the same for the dialetheist. Thus, for consistentists and dialetheists alike, it seems that the variants of the open pair present instances of semantic pathology that remain both undiagnosed and untreated.

Building up to Sorensen's solution, we begin with a brief discussion of Goldstein's attempt to resolve the open pair. We then explain the merits of Sorensen's view over Goldstein's, before posing a revenge problem that thwarts Sorensen's. We close with a critique of Priest's dialetheic response to the open pair, along with further consideration of how the failure of the truthmaker-gap approach to the open pair bears on Sorensen's larger project.

2 Sorensen (2001, pp. 181-2) endorses Goldstein's (1985) and (1992) truth-value gaps solution to the liar paradox, though he explicitly rejects Goldstein's (1992) application of this approach to the open pair. 


\section{Goldstein on the Open Pair}

Goldstein (1992, p. 2) views the open pair as a case of semantic pathology that underwrites an argument for truth-value gaps. ${ }^{3}$ In order to establish the gappiness of (1) and (2), Goldstein relies on two assumptions. Call the first the divergence assumption:

(DA) If each statement in [the open pair] has a unique truth-value, then each has the opposite value of the other. ${ }^{4}$

(DA) follows from a demand for consistency, since ascribing (1) and (2) the same truth-value yields inconsistency.

The second assumption derives from the most notable aspect of the open pair: the symmetry between (1) and (2). As each sentence says of the other exactly what the other says of it, any reason for giving one of those sentences a particular truth-value would seem equally to be reason for giving the other sentence the same truth-value. Goldstein takes these considerations to yield (what we will call) the symmetry assumption:

(SA) If each statement in [the open pair] has a unique truth-value, then each has the same value as the other. ${ }^{5}$

Combining (DA) with (SA), if the sentences in the open pair have unique truth-values, then they have both the opposite and the same truth-values. As Goldstein rejects inconsistency, he concludes that the antecedent of both (DA) and (SA) is false and thus that each sentence suffers from a truth-value gap. Let us call this the gappist solution to the open pair.

As a gappist solution to the simple liar (e.g., 'This sentences is false') is challenged by a potential re-emergence of semantic paradox in the form of the strengthened liar (e.g., 'This sentence is not true'), the gappist solution to the open pair is threatened by a potential re-emergence of the initial problem in the form of the strengthened open pair:

(3) (4) is not true

(4) (3) is not true.

As with the strengthened liar, positing gaps for (3) and (4) appears to yield inconsistency, since, if they are neither true nor false, it follows that they are

\footnotetext{
Goldstein takes his argument to motivate a gappist approach to "all the statements in Liartype paradoxes," $(1992$, p. 2) but since he refers to the truth-teller as a "variant of the Liar" we assume he includes it and the open pair in this class. We therefore take his point to be a general one about semantic pathology.

4 Goldstein (1992), p. 2.

5 Ibid. Goldstein here expands slightly on Buridan's reasons for assuming something like (SA) - see Hughes (1982, p. 73).
} 
not true, from which one is tempted to conclude that they are, therefore, true, after all.

Goldstein's response to strengthened versions of the open pair and the liar is to explain their gappiness as deriving from meaninglessness - from the failure of some tokens of these sentences to express propositions. ${ }^{6}$ While we are suspicious of the meaningless strategy, as a general resolution of semantic pathology, the issue is irrelevant here, for Goldstein's solution fails to diagnose - and, thus, to treat - the semantic pathology of the open pair.

To see why, consider the following case, which we call the asymmetric open pair:

(5) (6) is false

(6) (6) is false $\rightarrow(5)$ is false.

As in the earlier cases, (5) and (6) yield inconsistency, if we ascribe them the same truth-value; and they yield indeterminacy, if we demand a consistent ascription of truth-values. In contrast with the earlier cases, however, these sentences are not symmetric. There is thus no reason to endorse (SA) here, and, therefore, no reason to think that (5) and (6) have the same truth-value. Assuming consistency, however, (DA) still applies, and so still requires that we ascribe these sentences divergent truth-values, if any. This leaves Goldstein with indeterminacy, since, as with the (symmetric) open pairs, nothing favors one divergent ascription of truth-values over the other. As (SA) does not interact with (DA) in this case, Goldstein's argument for truthvalue gaps does not apply here, and so does not block the apparent indeterminacy of (5) and (6). Thus, modulo Goldstein's solution, the asymmetric open pair presents an instance of semantic pathology that Goldstein's appeal to truth-value gaps fails to resolve.

\section{Sorensen on the Open Pair}

Sorensen, like Goldstein, wishes to resolve the semantic pathology of the open pair, while retaining consistency and standard logic. Unlike Goldstein, he proposes a truthmaker, as opposed to a truth-value, gap, where a truthmaker gap obtains when a true sentence is not made true by anything else in the world. Postulating a truthmaker gap maintains appearances - that these sentences are not "truth-bearer illusions"- while responding to the problem the open pair presents.

Sorensen diagnoses the open pair as semantically determinate though epistemically indeterminate: Although the sentences of the open pair have unique, divergent truth-values, we cannot know which particular truth-value

\footnotetext{
Goldstein (1985) and (1992).

See Armour-Garb (2001).
} 
each has, since the absence of any truthmaker here denies us access to their truth-values. Moreover, since nothing makes the true sentence in the open pair true, it is pointless to note that anything that would make one true would equally make the other true as well. Since (SA) is, thus, rejected, we are free to conclude that these symmetric sentences have divergent truthvalues, as consistency and (DA) demand.

As this approach already denies the apparent force of symmetry, the version of the open pair that thwarts Goldstein's reliance on (SA) poses no challenge for Sorensen. In fact, the existence of the asymmetric case can be seen to strengthen Sorensen's position, for it can consistently resolve all of the versions of the open pair considered at this point, including the case that appears to undermines Goldstein's solution, in light of the latter's reliance on symmetry.

Thus far, Sorensen's approach to the open pair has fared well. But the truthmaker-gap solution quickly comes under strain, when confronted with its own revenge problems. In the case of the liar, the standard recipe for cooking up a revenge problem is to challenge a proposed solution by reformulating the paradox in terms of what the solution claims. Following this procedure, we get the following variant of the open pair:

(7) (8) has no truthmaker

(8) (7) has no truthmaker. ${ }^{8}$

If (7) and (8) were both false then each would have a truthmaker and, thus, would be true. So, to maintain consistency, they cannot both be false. Ascribing divergent truth-values to the sentences staves off inconsistency, but, as before, there are two ways of doing this, with nothing favoring one over the other. The problem for Sorensen is that if one of these sentences is true and the other false, then the true one- whichever it is - has a truthmaker. But it is utterly indeterminate which of the two sentences is the true one. Of course, Sorensen can consistently claim that both (7) and (8) are true-and, as such, without truthmakers - but this appears to undermine the motivation for positing truthmaker gaps in the first place, since they were introduced as a means for resolving, consistently, the indeterminacy of both the open pair and the sorites. ${ }^{9}$

In response, someone might, on Sorensen's behalf, attempt to marshal a special appeal to symmetry, in order to resolve the case of (7) and (8) - an

Thanks to Roy Sorensen for pushing us to discuss this case.

Sorensen could also claim that (7) and (8) are both meaningless, but this seems ad hoc and appears to work against minimizing truth-bearer illusions. 
appeal that applies to neither of (1) and (2) or (3) and (4). ${ }^{10}$ It is not clear how this would go, but how it would go is irrelevant, for, following the pattern of (5) and (6), we can generate an asymmetric case with exactly the same features as (7) and (8):

\section{(9) (10) has no truthmaker}

(10) (10) has no truthmaker $\rightarrow$ (9) has no truthmaker.

Here too, the only truth-value ascriptions that comport with a truthmaker-gap approach are to take (9) and (10) both to be true (and thus without truthmakers). However, nothing motivates this ascription over a divergent one, nothing, that is, apart from wanting to avoid an indeterminacy that cannot be accounted for with truthmaker gaps.

While Sorensen's approach can ascribe truth-values to the cases just considered, they break the connection between indeterminacy and truthmaker gaps. To drive the wedge between them further, consider what we will call the truthmaker open pair:

(11) (12) is true $\rightarrow$ ((11) is false \& (12) has no truthmaker)

(12) (11) is true $\rightarrow\left((12)\right.$ is false $\&$ (11) has no truthmaker). ${ }^{11}$

Working through the possible truth-values, consistency demands divergent truth-values for (11) and (12)-one is true and the other is false-with nothing to favor one divergent set of ascriptions over the other. Thus, (11) and (12) give rise to indeterminacy.

That said, as a brief consideration makes clear, (11) and (12) undermine any attempt to resolve their semantic pathology consistently, by classifying the resulting indeterminacy as merely epistemic. To see why, ascribe truth to (11) and falsity to (12). Since the first conjunct of (11)'s consequent would make the consequent false, (11)'s antecedent must be false, which (ex hypothesi) it is. The antecedent of (12) is true (ex hypothesi), so for this sentence to be false its consequent must be false. Since the first conjunct of

10 While not something Sorensen is likely to endorse, someone might consider this tactic on the basis of Sorensen's (2003) discussion of what he calls the definite no-no paradox, viz., an open pair case that employs 'not definitely true' instead of 'false'. This case too is one where both sentences can be true (though not definitely true or untrue). Here Sorensen notes, "Symmetry precludes one from being true while the other is false" (p. 228). Combining an appeal to symmetry with a demand for consistency, then, would rule out all truth-value ascriptions except 'true' for both sentences in the pair. Whether this tactic would work when applied to (7) and (8) (or, indeed, to the definite no-no paradox) is an open question, but it is not one that we can cover here.

11 We take this case to form a version of what we call the curried open pair, on the model of Curry's paradox. Replacing the consequents of (11) and (12) with the absurdity constant ' $\perp$ ', the resulting pair yields indeterminacy, inconsistency, or trivialism. 
this consequent is true (again, ex hypothesi), the second must be false. That is, it must be false that (11) has no truthmaker, from which (via fiddling) we conclude that (11) has a truthmaker. Thus, we can consistently maintain that (11) is true and (12) is false, provided we also maintain that (11) has a truthmaker. A parallel argument shows that we can maintain that (11) is false and (12) is true, provided we also maintain that (12) has a truthmaker.

There is an indeterminacy here, to be sure, but Sorensen cannot explain it in terms of a truthmaker gap, since the only way to avoid contradiction is to maintain that the true sentence in this pair-whichever it is-possesses a truthmaker. The problem cannot be simply that a truthmaker gap deprives us of access to the, in fact, determinate truth-values of (11) and (12), for what is indeterminate in this case is which of these sentences some truthmaker makes true. Hence, there is a variant of the open pair that Sorensen cannot resolve via an appeal to truthmaker gaps and thus a kind of indeterminacy that he cannot characterize as epistemic.

Again, as a last resort, someone might, on Sorensen's behalf, try to motivate a special appeal to symmetry of the sort mentioned above, in order to class (11) and (12) as inconsistent and thus with neither truthmakers nor truth-values. It is unlikely Sorensen would endorse this strategy, given his interests, but, in any case, it would not help, for consider what we call the asymmetrical truthmaker open pair:

(13) (14) is true $\rightarrow$ ((13) is false \& (14) has no truthmaker)

(14) [(14) is true $\rightarrow((13)$ is false \& (14) has no truthmaker)] $\rightarrow$ [(13) is true $\rightarrow((14)$ is false \& (13) has no truthmaker)].

As with (5) and (6), there appears to be no compelling reason for ascribing (13) and (14) the same truth-value and, thus, no reason for thinking that the resulting inconsistency necessitates truth-value gaps. But maintaining consistency demands divergent truth-value ascriptions to (13) and (14), which, in turn, both yields indeterminacy and, as is easy to verify, the existence of an indeterminate truthmaker.

This, in turn, casts doubt on Sorensen's ability to resolve any version of the open pairs. After all, one lesson we have learned from attempts to solve the liar paradox is that, to be adequate, a solution must ramify about all versions of that paradox. By extension, to be adequate, a resolution of the less familiar instances of semantic pathology that the open pair presents must resolve all variants of this case. Sorensen does not meet this condition of adequacy, thereby leaving the semantic pathology of the open pair both undiagnosed and untreated. 


\section{Concluding Remarks}

As we have argued, Sorensen, like Goldstein, fails to resolve the semantic pathology that plagues variants of the open pair, while maintaining consistency and classical logic. What conclusion should we draw at this point? Priest, in his contribution to this symposium, argues that the open pair requires a dialetheic resolution. He criticizes Sorensen's rejection of symmetry in the case of the open pair, claiming that, even if one denies the truthmaker principle, maintaining that (1) and (2) have different semantic properties is "a manifest a priori repugnance" (Priest (2004), p. 6). Ascribing them matching truth-values avoids indeterminacy, but, as noted above, if these sentences have the same truth-value, then each is both true and false. ${ }^{12}$ Thus, as Goldstein sees his solution to the open pair as grist for the meaninglessness of liar sentences, generally, and Sorensen sees his solution to the open pair as grist for his epistemicist solution to the sorites, Priest may see his inconsistent solution to the open pair as further support for dialetheism.

While this dialetheic strategy might appear successful, as applied to the open pair, and even to the truthmaker open pair, the symmetry-breaking cases show that a dialetheist cannot even inconsistently resolve the semantic pathology of all variants of the open pair. For, although the dialetheist can ascribe the same truth-value to (5) and (6), (9) and (10), or (13) and (14), he can also consistently ascribe them divergent truth-values, with no apparent reason for favoring any matching or divergent assignment over any other. Thus, the indeterminate semantic pathology of variants of the open pair is impartial, appearing to plague both consistentists and inconsistentists, alike.

As with Sorensen's approach, the inadequacy of the dialetheic response to the asymmetrical cases of the open pair casts doubt on its ability to handle the other, symmetrical ones. The aforementioned condition of adequacy thus appears to render the dialetheist unable to resolve the semantic pathology of any variants of the open pair.

In closing, we should note that, per Sorensen, "the truthmaker gap solution to the [open pair] is a precedent for an epistemic solution to the sorites paradox" (p. 176). Insofar as his attempt to resolve the open pair fails, his approach to the latter problem requires re-thinking as well. ${ }^{13}$ At the very least, the open pair re-presents a challenge to those, such as Sorensen, Gold-

12 Against a gappist response (e.g., Goldstein's) to the upshot of symmetry, Priest (2004), fn. 3 argues that an ascription of gaps leads to an assignment of both gaps and gluts, so it is simpler, methodologically speaking, just to go with gluts.

13 While Priest rejects Sorensen's approach to the open pair, he agrees with Sorensen that the sorites and the open pair are of a piece. Although we will not pursue the issue here, it is clear that if Priest insists on this tie, his failure to resolve the open pair would raise questions about any solution he might offer for the sorites, as well. 
stein, and (in a different sense) Priest, who attempt to resolve-consistently or inconsistently - the oddity of this case of semantic pathology. ${ }^{14}$

\section{References}

Armour-Garb, B. (2001) "Deflationism and the Meaningless Strategy." Analysis 61: 280-89.

Goldstein, L. (1985) "The Paradox of the Liar-a Case of Mistaken Identity." Analysis 45: 9-13.

Goldstein, L. (1992) “"This Statement is Not True' is Not True." Analysis 52: $1-5$.

Hughes, G. (1982) John Buridan on Self-Reference. Cambridge: Cambridge University Press.

Priest, G. (2004) "Words without Knowledge." Philosophy and Phenomenological Research (Forthcoming).

Sorensen, R. (2001) Vagueness and Contradiction. Oxford: Clarendon Press.

Sorensen, R. (2003) “A Definite No-No.” In Liars and Heaps (pp. 225-229), ed. JC Beall. Oxford: Oxford University Press.

14 Thanks to Roy Cook, Laurence Goldstein, Graham Priest, and Roy Sorensen, for helpful discussion. 